Corresponding author: jaster@ partners.org

C) 2015 Knoechel et al. This article is distributed under the terms of the Creative Commons Attribution License, which permits unrestricted reuse and redistribution provided that the original author and source are credited.

Ontology terms: leukemia

Published by Cold Spring Harbor Laboratory Press

doi: $10.1101 /$ mcs.a000539

\section{Complete hematologic response of early T-cell progenitor acute lymphoblastic leukemia to the $\gamma$-secretase inhibitor BMS-906024: genetic and epigenetic findings in an outlier case}

Birgit Knoechel, ${ }^{1,9}$ Ami Bhatt, ${ }^{2,9}$ Li Pan, ${ }^{3}$ Chandra S. Pedamallu, ${ }^{1,4}$ Eric Severson, ${ }^{3}$ Alejandro Gutierrez, ${ }^{5}$ David M. Dorfman, ${ }^{3}$ Frank C. Kuo, ${ }^{3}$ Michael Kluk, ${ }^{3}$ Andrew L. Kung, ${ }^{6}$ Patrick Zweidler-McKay, ${ }^{7}$ Matthew Meyerson, ${ }^{1,3,4}$ Stephen C. Blacklow, ${ }^{8}$ Daniel J. DeAngelo, ${ }^{1}$ and Jon C. Aster ${ }^{3}$

\footnotetext{
${ }^{1}$ Department of Medical Oncology, Dana-Farber Cancer Institute, Boston, Massachusetts 02215, USA; ${ }^{2}$ Departments of Medicine and Genetics, Stanford University, Stanford, California 95305, USA; ${ }^{3}$ Department of Pathology, Brigham and Women's Hospital, Boston, Massachusetts 02115, USA; ${ }^{4}$ Broad Institute of MIT and Harvard University, Cambridge, Massachusetts 02142, USA; ${ }^{5}$ Division of Hematology/Oncology, Boston Children's Hospital and Dana-Farber Cancer Institute, Boston, Massachusetts 02115, USA; ${ }^{6}$ Department of Pediatrics, Columbia University Medical Center, New York, New York 10032, USA; ${ }^{7}$ Department of Pediatrics, University of Texas MD Anderson Cancer Center, Houston, Texas 77030, USA; ${ }^{8}$ Department of Biochemistry and Molecular Pharmacology, Harvard Medical School, Boston, Massachusetts 02115, USA
}

Abstract Notch pathway antagonists such as $\gamma$-secretase inhibitors (GSIs) are being tested in diverse cancers, but exceptional responses have yet to be reported. We describe the case of a patient with relapsed/refractory early T-cell progenitor acute lymphoblastic leukemia (ETP-ALL) who achieved a complete hematologic response following treatment with the GSI BMS-906024. Whole-exome sequencing of leukemic blasts revealed heterozygous gain-of-function driver mutations in NOTCH1, CSF3R, and PTPN11, and a homozygous/ hemizygous loss-of-function mutation in DNMT3A. The three gain-of-function mutations were absent from remission marrow cells, but the DNMT3A mutation persisted in heterozygous form in remission marrow, consistent with an origin for the patient's ETPALL from clonal hematopoiesis. Ex vivo culture of ETP-ALL blasts confirmed high levels of activated NOTCH1 that were repressed by GSI treatment, and RNA-seq documented that GSIs downregulated multiple known Notch target genes. Surprisingly, one potential target gene that was unaffected by GSIs was MYC, a key Notch target in GSI-sensitive TALL of cortical T-cell type. H3K27ac super-enhancer landscapes near MYC showed a pattern previously reported in acute myeloid leukemia (AML) that is sensitive to BRD4 inhibitors, and in line with this ETP-ALL blasts downregulated MYC in response to the BRD4 inhibitor JQ1. To our knowledge, this is the first example of complete response of a Notch-mutated ETP-ALL to a Notch antagonist and is also the first description of chromatin landscapes associated with ETP-ALL. Our experience suggests that additional attempts to target Notch in Notch-mutated ETP-ALL are merited.

[Supplemental material is available for this article.]

\footnotetext{
${ }^{9}$ These authors contributed equally to this work.
} 


\section{INTRODUCTION}

The Notch signaling pathway plays a key role in early stages of normal T-cell development and when hyperactivated is a potent inducer of T-cell acute lymphoblastic leukemia (T-ALL) (for recent review, see Van Vlierberghe and Ferrando 2012). The most common mechanism for oncogenic activation of Notch signaling in T-ALL is gain-of-function mutations in NOTCH1, which occur in $>50 \%$ of human T-ALLs. T-ALLs can be subclassified into early T-cell progenitor ALL (ETP-ALL), cortical T-ALL, and mature T-ALL based on stage-specific differentiation markers, with ETP-ALLs being defined by the absence of CD4, CD8, and CD1a and frequent expression of one or more myeloid markers (Coustan-Smith et al. 2009). Early work suggested that NOTCH1 mutations were absent from ETP-ALL; indeed, T-ALLs expressing myeloid markers, a characteristic of ETP-ALL, have been excluded from some trials of Notch pathway inhibitors. However, earlier sequencing studies focused on pediatric ETP-ALL (Zhang et al. 2012), and more recent studies of adult ETP-ALL have shown that NOTCH1 mutations occur in a significant minority of cases (Neumann et al. 2013).

Notch receptors are large multimodular single-pass transmembrane proteins that are activated when they engage ligands of the JAGGED or DELTA families expressed on neighboring cells (for review, see Kopan and llagan 2009). Ligand binding alters the conformation of a juxtamembrane negative regulatory region (NRR), rendering Notch sensitive to successive cleavages by ADAM metalloproteases and the intramembranous $\gamma$-secretase complex. $\gamma$-Secretase cleavage in turn allows the intracellular portion of Notch (ICN) to translocate to the nucleus and form a transcription activation complex with the DNA-binding factor RBPJ and coactivators of the MAML family. The most common NOTCH1 mutations in T-ALL derange the structure of the NRR (Malecki et al. 2006), leading to ligand-independent ICN generation and overexpression of key target genes such as MYC, mainly via interaction of ICN/RBPJ complexes with long-range enhancers (Herranz et al. 2014; YashiroOhtani et al. 2014).

The frequent presence of NOTCH1 gain-of-function mutations in T-ALL has provided the rationale for clinical trials of GSIs. Initial trials were plagued by on-target gut toxicity, and the perception that GSI have unacceptable toxicities is still pervasive. However, with intermittent dosing, GSIs can be given safely and have tolerable toxicities, as shown by phase I trials of GSIs in solid tumors (for review, see Aster and Blacklow 2012). What mainly has been lacking is evidence of strong sustained anti-tumor responses. Here we describe the case of a 53-yr-old male with relapsed refractory ETP-ALL who experienced a complete hematologic remission associated with a deep molecular response following treatment with the GSI BMS-906024.

\section{RESULTS}

A male patient presented at the Dana-Farber Cancer Institute (DFCl) at the age of $53 \mathrm{yr}$ with relapsed refractory ETP-ALL. ETP-ALL was first diagnosed 8 mo prior to presentation. At this time, flow cytometric analysis showed a CD45(dim) blast population that was positive for CD34 (84\%), CD3 (cytoplasmic, 90\%), CD5 (75\%), CD7 (86\%), CD33 (87\%), and TdT(dim, $58 \%$ ), and negative for CD1a, CD4, and CD8. Cytogenetics revealed a 46,XY karyotype with del(7p13). The patient was treated with induction chemotherapy at an outside institution per the CALGB 9111 regimen (Larson et al. 1998). A repeat marrow examination 4 wk after induction was interpreted as being consistent with remission, and repeat cytogenetic analysis showed a normal 46,XY karyotype. After two cycles of consolidation chemotherapy, the patient was started on methotrexate/mercaptopurine maintenance therapy $6.5 \mathrm{mo}$ after 
initial presentation. This was complicated by severe neutropenia, necessitating a $50 \%$ reduction in the second cycle of maintenance chemotherapy.

A routine follow-up marrow examination before the third cycle of maintenance therapy showed evidence of early relapse of ETP-ALL, and he was referred to the DFCI for further treatment. Two cycles of salvage nelarabine therapy were given, but repeat marrow examination after the second cycle revealed persistent ETP-ALL. Flow cytometric analysis at this time showed CD45(dim) blasts that were positive for CD34 (99\%), HLA-DR (10\%), CD5 (97\%), CD7 (98\%), CD13 (98\%), CD33 (100\%), and TdT (dim, small subset), and negative for CD1a, CD4, CD8, and surface CD3. Cytogenetic analysis again showed a 46,XY karyotype with del(7p13). TCR (encoded by TRG) gene analysis revealed monoallelic rearrangement of TCRy (data not shown), a feature also consistent with ETP-ALL that has been associated with high-risk disease (Gutierrez et al. 2010).

The patient was enrolled on a trial of BMS-906024, an intravenously administered GSI, at a dose of $6 \mathrm{mg}$ once per week in 4-wk cycles. The patient also received dexamethasone, $20 \mathrm{mg}$ orally, for $4 \mathrm{~d}$ with the first dose of BMS-906024 during the first and second cycles of BMS-906024 treatment. The first 4-wk cycle of BMS-906024 was complicated by severe thrombocytopenia, which necessitated holding BMS-906024 for 1 wk after the second dose, after which treatment resumed. By the end the first 4-wk cycle of therapy, blasts were cleared from the peripheral blood (Table 1) and bone marrow (Fig. 1). By the end of the second 4-wk cycle of BMS-906024, peripheral blood counts had normalized (Table 1), the marrow was normocellular and showed maturing trilineage hematopoiesis (Fig. 1), and minimal residual disease testing by flow cytometry was negative $\left(1 \times 10^{6}\right.$ events evaluated). Cytogenetic analysis revealed a normal 46,XY karyotype.

The patient subsequently received one additional 4-wk cycle of BMS-906024 without complication, and then was removed from protocol in preparation for hematopoietic stem cell transplantation from a matched unrelated donor. Conditioning for transplantation consisted of Cytoxan, $1800 \mathrm{mg} / \mathrm{m}^{2}$, for $2 \mathrm{~d}$ and $1200 \mathrm{cGy}$ of total body irradiation in six fractionated doses. The posttransplant course was complicated by grade 4 skin and ocular acute graft-versus-host disease (GVHD) and subsequently by moderately severe skin and ocular chronic GVHD. He is now 19 mo posttransplant with no evidence of leukemia.

To gain insight into the basis of this tumor's response to BMS-906024, we initially performed targeted next-generation sequencing (NGS) using a previously reported gene panel (Wagle et al. 2012) and subsequently extended this to whole-exome NGS (WES). Both sequencing analyses revealed driver mutations in NOTCH1, PTPN11, and DNMT3A, and WES revealed an additional driver mutation in CSF3R (Table 2), a gene that was not covered

\begin{tabular}{lccccccccccccccc}
\hline \multicolumn{10}{l}{ Table 1. Hematologic response following initiation of BMS-906024 treatment } \\
\hline Days & 0 & 7 & 14 & 21 & 28 & 35 & 42 & 50 & 56 & 63 & 76 & 83 & 101 \\
\hline GSI & + & + & & + & + & + & + & + & + & + & + & & \\
DEX & + & & & & + & & & & & & & & \\
WBC & 46 & 14.7 & 9.3 & 0.9 & 0.9 & 1.6 & 1.6 & 2.2 & 3.9 & 4.1 & 6.3 & 8.9 & 6.4 \\
Blasts & $85 \%$ & $80 \%$ & $46 \%$ & - & - & - & - & - & - & - & - & - & - \\
Hgb & 9.9 & 10.8 & 10.1 & 7.4 & 8.5 & 10.5 & 8.9 & 10.8 & 11.3 & 11.8 & 12 & 12.9 & 12 \\
Plt & 42 & 13 & 7 & 4 & 9 & 22 & 50 & 91 & 117 & 109 & 179 & 166 & 163 \\
MRD & & & & & Neg* & & & & Neg** & & & & \\
\hline
\end{tabular}

GSI, days on which BMS-906024 was administered; DEX, starting point of $4 \mathrm{~d}$ cycles of dexamethasone; WBC, white blood cell count, $10^{3} / \mathrm{mm}^{3}$; blasts, peripheral blood blasts; Hgb, hemoglobin, gm/dL; Plt, platelet count, $10^{3} / \mathrm{mm}^{3}$; MRD, minimal residual disease by flow cytometry; Neg*, no leukemic blasts detected in $1.2 \times 10^{5}$ events; Neg**, no leukemic blasts detected, $1 \times 10^{6}$ events. 

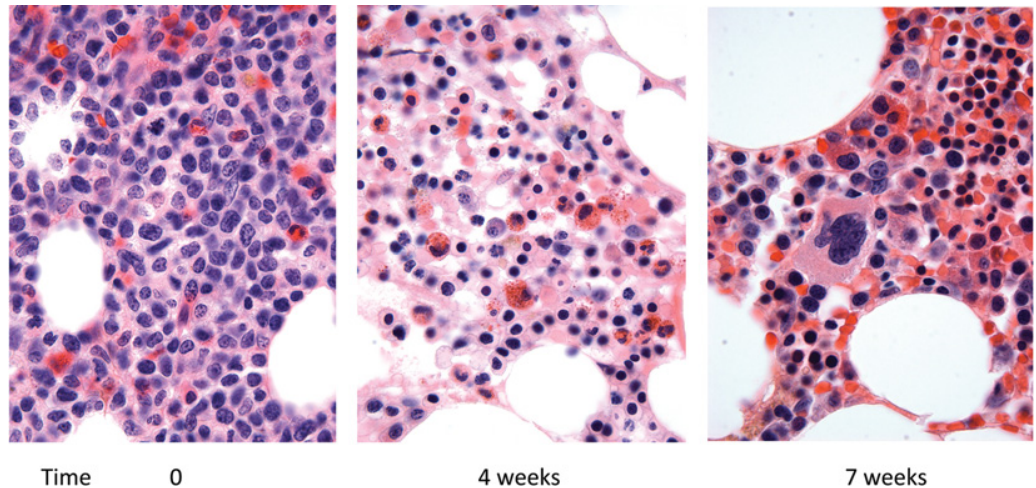

Figure 1. Bone marrow biopsy histology prior to and 4 and $7 \mathrm{wk}$ after initiation of treatment with BMS-906024 (hematoxylin and eosin staining, 400× magnification). The marrow contains mainly blasts prior to treatment that are cleared following treatment.

by the targeted gene panel used. All four driver mutations were subsequently confirmed by direct Sanger sequencing of PCR products (Supplemental Fig. 1). Based on the variant allele fraction, the CSF3R mutation was present at heterozygous dosage and resulted in a T618I substitution. This mutation has been described in a high fraction of chronic neutrophilic leukemia and produces constitutive activation of CSF3R (also known as the G-CSF receptor) (Maxson et al. 2013). The PTPN11 mutation also appeared to be heterozygous based on variant allele fraction and resulted in a F285S substitution that corresponds to a gain-of-function mutation implicated in a subset of Noonan syndrome (Tartaglia and Gelb 2005). Virtually all DNMT3A sequence reads were mutant; based on lack of evidence of copy-number variation in the region of Chromosome 1 containing DNMT3A (described below), this mutation appears to be homozygous and creates a nonsense mutation at codon 402. The encoded mutated polypeptide, DNMT3A Q402*, lacks the carboxy-terminal

Table 2. Genomic alterations identified by next-generation sequencing

\begin{tabular}{|c|c|c|c|c|c|c|}
\hline Gene & Chrom. & $\begin{array}{c}\text { HGVS } \\
\text { DNA Ref. }\end{array}$ & $\begin{array}{l}\text { HGVS } \\
\text { protein ref. }\end{array}$ & $\begin{array}{l}\text { Variant allele } \\
\text { fraction (\%) }\end{array}$ & $\begin{array}{c}\text { Fold } \\
\text { coverage }\end{array}$ & Effect \\
\hline \multicolumn{7}{|c|}{ Targeted-exon sequencing (leukemia, bone marrow aspirate, $85 \%$ blasts) } \\
\hline NOTCH1 & 9 & c. $4775 \mathrm{~T}>\mathrm{G}$ & p.F1592C & 64.9 & 165 & GOF \\
\hline PTPN11 & 12 & c. $854 \mathrm{~T}>\mathrm{C}$ & p.F285S & 40.0 & 115 & GOF \\
\hline DNMT3A & 2 & c. $1204 C>T$ & p.Q402* & 99.3 & 139 & LOF \\
\hline \multicolumn{7}{|c|}{ Whole-exome sequencing (leukemia, bone marrow aspirate, 85\% blasts) } \\
\hline NOTCH1 & 9 & c. $4775 \mathrm{~T}>\mathrm{G}$ & p.F1592C & 70.5 & 94 & GOF \\
\hline PTPN11 & 12 & c. $854 \mathrm{~T}>\mathrm{C}$ & p.F285S & 45.7 & 142 & GOF \\
\hline DNMT3A & 2 & c. $1204 C>T$ & p.Q402* & 98.7 & 89 & LOF \\
\hline CSF3R & 1 & c. $1853 C>T$ & pT6181 & 46.2 & 97 & GOF \\
\hline \multicolumn{7}{|c|}{ Whole-exome sequencing (remission marrow, no identified lymphoblasts) } \\
\hline NOTCH1 & 9 & WT & & & 48 & \\
\hline PTPN11 & 12 & WT & & & 108 & \\
\hline DNMT3A & 2 & c. $1204 C>T$ & p.Q402* & 54.6 & 67 & LOF \\
\hline CSF3R & 1 & WT & & & 76 & \\
\hline
\end{tabular}

HGVS, Human Genome Variation Society; GOF, gain of function; LOF, loss of function; WT, wild type. 
plant homeodomain and catalytic domain that are required for DNMT3A function. Consistent with the diagnosis of ETP-ALL, gain-of-function CSF3R and PTPN11 mutations and loss of function DNMT3A mutations have been described in ETP-ALL (Coustan-Smith et al. 2009; Zhang et al. 2012; Maxson et al. 2013), but not, to the best of our knowledge, in cortical or mature T-ALL.

The NOTCH1 mutation is predicted to create a F1592C substitution in the NOTCH1 negative regulatory region (NRR) (Fig. 2A), which is the most common site of NOTCH1 gain-offunction mutations in T-ALL and ETP-ALL. Because the F1592C mutation has not been described, we scored this mutant in functional studies using a standard Notch reporter gene assay in which NRR mutants are expressed in a form of NOTCH1 lacking the ligand-binding region of the receptor (Malecki et al. 2006), enabling measurement of the effects of various sequence variants on ligand-independent NOTCH1 activation. This assay confirmed that F1592C causes ligand-independent $\gamma$-secretase-dependent activation of NOTCH1 signaling

A

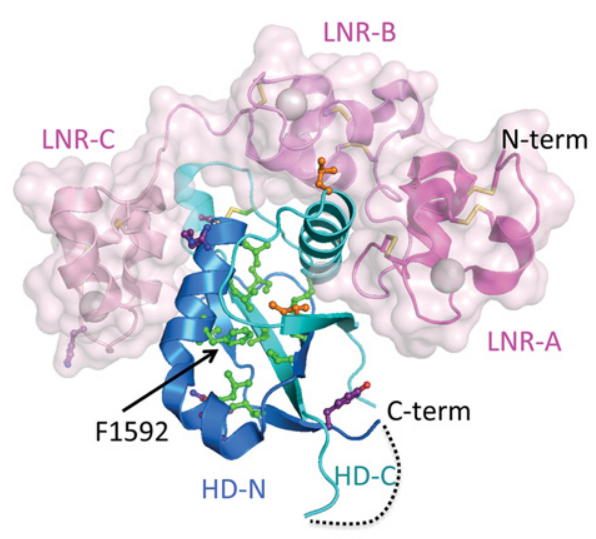

C

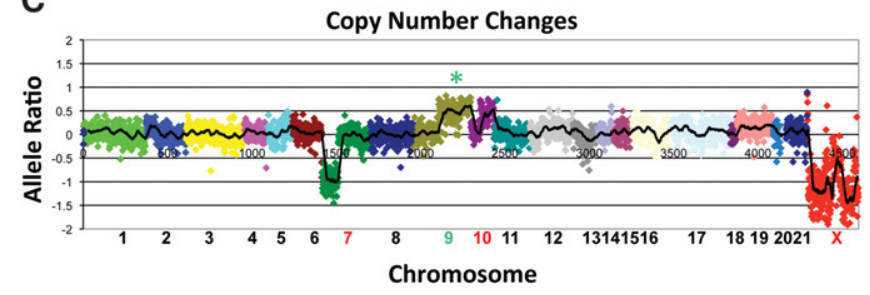

B

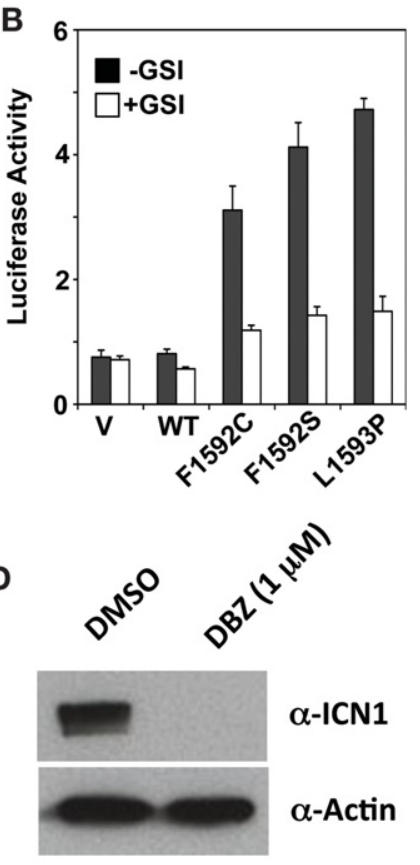

Figure 2. Characterization of the NOTCH1 gain-of-function mutation F1592C. (A) Position of F1592C in the $\mathrm{NOTCH} 1$ negative regulatory region (NRR). Mapping is based on the crystallographic structure of Gordon et al. (2009b). Residues previously reported be mutated in T-ALL are shown in green (core residues), orange (interdomain interface residues), or purple (polar residues). The three LNR repeats are colored various shades of pink with a translucent surface, and the core "heterodimerization domain" is colored blue prior to the site of furin cleavage (HD-N) and cyan thereafter (HD-C). F1592 sits in the center of the hydrophobic core of the heterodimerization domain. The image is rendered with the program Pymol and is adapted from Gordon et al. (2009a). (B) F1592C leads to ligand-independent activation of NOTCH1. The ability of the F1592C variant to activate a Notch firefly luciferase reporter gene was compared with wild-type NOTCH1 and two previously characterized gain-of-function mutants, F1592S and L1593P. Firefly luciferase activity was normalized to an internal Renilla luciferase control reporter gene. Normalized firefly luciferase activity is expressed related to the activity of wild-type NOTCH1, which is arbitrarily set to a value of 1. Each expression plasmid was tested in three independent experiments; error bars represent the standard deviations. $(C)$ Copy-number gains and losses. The copy number of chromosomal regions was determined from sequencing data as described (Wagle et al. 2012). (D) Effect of the GSI diaminobenzidine (DBZ) on activated NOTCH1 (ICN1) levels in leukemic blasts. Blasts were treated with vehicle (DMSO [dimethyl sulfoxide]) or DBZ for $3 \mathrm{~d}$ prior to harvest and preparation of whole-cell lysates. A representative Western blot stained for activated NOTCH1 (ICN1) and $\beta$-actin is shown. 
(Fig. 2B). We also used the NGS data to determine genomic copy-number changes. This revealed the $7 p$ deletion noted by karyotyping and a previously unrecognized 10p deletion and a single copy gain involving $9 q$, including the region encompassing the NOTCH1 locus (Fig. 2C), an event that has been reported in T-ALL (van Vlierberghe et al. 2006). In this case, the $9 q$ duplication involved the mutated NOTCH1 allele, as the variant allele:WT allele read ratio was $\sim 2: 1$ in both sequencing analyses (Table 2). In line with these observations, studies performed in vitro prior to initiation of BMS-906024 therapy showed that the leukemic blasts contained high levels of activated NOTCH1 (ICN1) that were markedly decreased by treatment with the GSI DBZ (Fig. 2D).

To further investigate the nature of this patient's tumor and its response to BMS-906024, we also performed WES on remission marrow obtained after cycle 2 of GSI. This showed wild-type reads for NOTCH1, PTPN11, and CSF3R, and persistence of the DNMT3A mutation at heterozygous dosage. To exclude the possibility of a germline heterozygous DNMT3A mutation we performed Sanger sequencing on buccal mucosal DNA obtained from the patient following allogeneic transplantation (Fig. 3A). This revealed only wildtype DNMT3A reads, in contrast to analyses done on DNA prepared from ETP-ALL blasts and remission marrow pretransplant, which confirmed the presence of homozygous and heterozygous DNMT3A codon 402 mutations, respectively (Fig. 3A). These findings are consistent with a scenario in which ETP-ALL arose out of a background of clonal hematopoiesis associated with a heterozygous DNMT3A mutation, a relationship revealed by clearance of ETP-ALL following treatment with BMS-906024 (Fig. 3B).

To identify likely NOTCH1 target genes, we next performed RNA-seq on blasts treated with GSI or vehicle (DMSO) (Fig. 4A). The genes that were most sensitive to GSI included multiple Notch target genes previously identified in cortical T-ALL cells, many of which are regulated by long-range enhancers (Wang et al. 2014). Surprisingly, MYC, an important Notch target gene in cortical T-ALL, was not downregulated by GSI treatment at the level of RNA or protein, as Western blotting showed no change in MYC protein despite a sharp decreased in ICN1 levels following GSI treatment (Fig. 4B). We and others recently described a complex multidomain enhancer region 3' of MYC (Herranz et al. 2014; YashiroOhtani et al. 2014). In cortical T-ALL cells in which MYC is a direct target of Notch, MYC
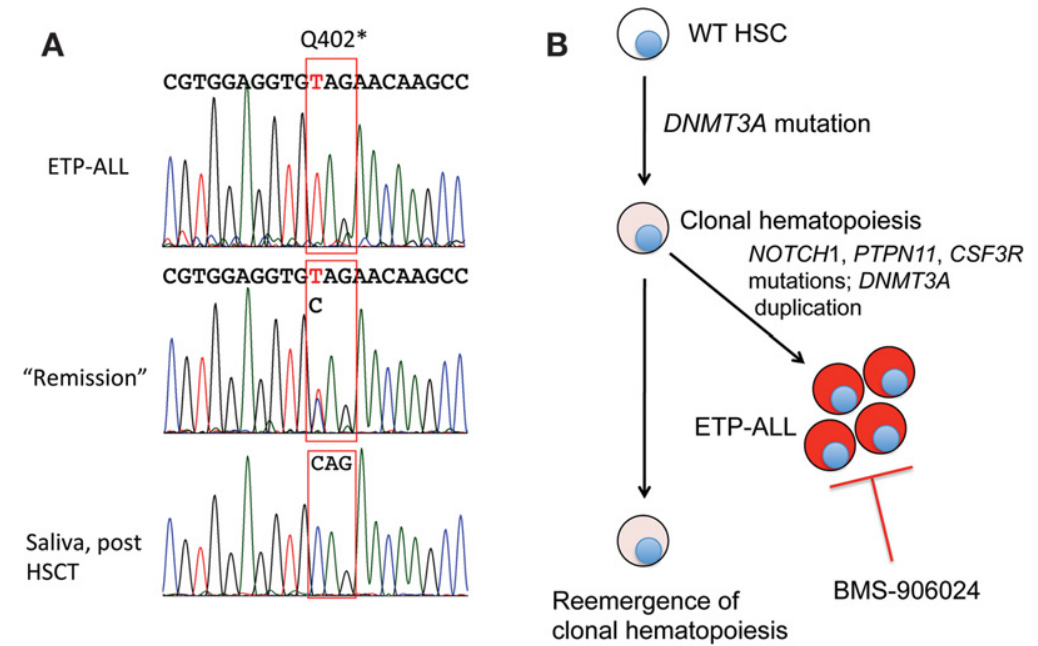

Figure 3. Evidence of ETP-ALL origin from clonal hematopoiesis. (A) Sanger sequencing traces of DNMT3A. Traces were obtained from DNA obtained from blasts prior to treatment, remission marrow, and saliva following hematopoietic stem cell transplantation. (B) Reconstructed clonal evolution of ETP-ALL, based on sequencing of DNMT3A as shown in A. 
A

\begin{tabular}{|l|l|l|l|}
\hline \multicolumn{1}{|c|}{ Gene } & $\begin{array}{c}\text { Control } \\
\text { FPKM }\end{array}$ & $\begin{array}{c}\text { GSI } \\
\text { FPKM }\end{array}$ & $\begin{array}{c}\text { Fold } \\
\text { Change }\end{array}$ \\
\hline HES1 & 3.93 & 0.32 & 12.2 \\
\hline HES4 & 3.35 & 0.93 & 5.4 \\
\hline MYO7B* & 5.65 & 3.65 & 4.0 \\
\hline TASP1 & 5.11 & 3.34 & 3.4 \\
\hline DTX1* & 1.13 & -0.23 & 2.6 \\
\hline GIMAP4* & 2.26 & 1.05 & 2.3 \\
\hline GIMAP5* & 4.64 & 3.28 & 2.6 \\
\hline GIMAP7* & 5.07 & 3.69 & 2.6 \\
\hline GIMAP8* & 2.65 & 1.37 & 2.4 \\
\hline LTZFL1 & 4.25 & 3.56 & 1.6 \\
\hline IGFR1* & 3.09 & 2.41 & 1.6 \\
\hline
\end{tabular}

C

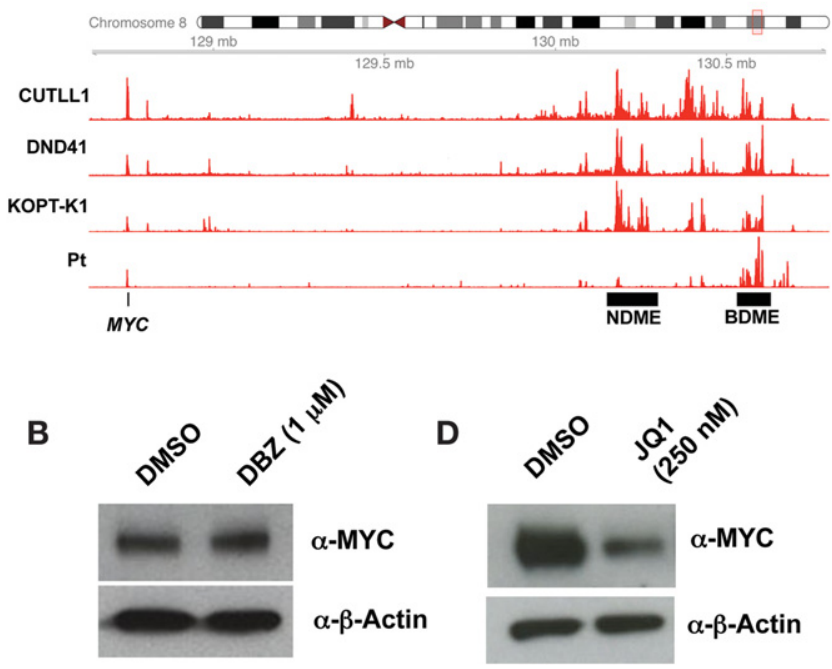

Figure 4. Characterization of likely Notch target genes and MYC in ETP-ALL. (A) Genes downregulated by the GSI DBZ (1 $\mu \mathrm{m})$ in ETP-ALL blasts. Cells were treated with DBZ or control (DMSO) for $3 \mathrm{~d}$ followed by harvest and analysis by RNA sequencing. FPKM represents the $\log _{2}$ value of the FPKM (fragments per transcript kilobase per million fragments mapped). (B) Effect of the GSI DBZ on MYC levels in leukemic blasts. Blasts were treated with vehicle (DMSO) or DBZ for $3 \mathrm{~d}$ prior to harvest and preparation of whole-cell lysates. A representative Western blot stained for MYC and $\beta$-actin is shown. (C) H3K27ac ChIP-seq landscapes near MYC. Aligned reads are shown for leukemic blasts (Pt) and three NOTCH1-mutated T-ALLs, DND41, KOPT-K1, and CUTLL1, that downregulate MYC in response to GSI treatment. (NDME) Notch-dependent MYC enhancer; (BDME) BRD4-dependent MYC enhancer. (D) Effect of the bromodomain inhibitor JQ1 on MYC levels in leukemic blasts. Blasts were treated with vehicle (DMSO) or JQ1 for $3 \mathrm{~d}$ prior to harvest and preparation of whole-cell lysates. A representative Western blot stained for MYC and $\beta$-actin is shown.

transcription is regulated by a conserved CSL/NOTCH1 binding site within the $3^{\prime}$ enhancer region that lies $\sim 1.3 \mathrm{Mb}$ from the MYC promoter. This enhancer element, termed the Notchdependent Myc enhancer (NDME), is required for thymocyte development and for induction of T-ALL by Notch in mice (Herranz et al. 2014). To understand the lack of responsiveness of MYC to Notch inhibition in this case, we performed H3K27ac chromatin immunoprecipitation sequencing (ChIP-seq) on blasts obtained prior to BMS-906024 treatment (Fig. 4C). This revealed that the NDME was inactive, being largely lacking in H3K27ac marks. In contrast, high levels of H3K27ac marks were observed in a more distal $3^{\prime}$ region termed the BRD4-dependent MYC enhancer (BDME), which has been implicated in regulation of MYC in acute myeloid leukemia (AML) (Shi et al. 2013) and in GSI-resistant cortical T-ALL (Yashiro-Ohtani et al. 2014). In line with these observations, MYC protein levels in patient blasts were downregulated by the BRD4 inhibitor JQ1 (Fig. 4D). Thus, the MYC enhancer state in this GSI-responsive ETP-ALL resembles that seen in AML rather than typical cortical-type T-ALL.

Additional work done to try to further evaluate the basis for the response to GSI in this case included attempts to engraft NSG (NOD scid $\gamma$ ) and NSG-S mice (NSG mice expressing IL-3, GM-CSF, and KIT ligand) with patient blasts administered by tail vein injection. No growth was observed in NSG mice. In contrast, leukemic blasts grew out in three of three NSG-S primary recipient mice 6 mo after injection (Fig. 5A). Immunohistochemistry revealed that the blasts had detectable ICN1 (Fig. 5B), Sanger sequencing of DNA prepared from leukemic blasts confirmed that the tumor maintained the same genotype (data not shown), and flow cytometry confirmed an immunophenotype consistent with ETP-ALL, as the blasts were 
A

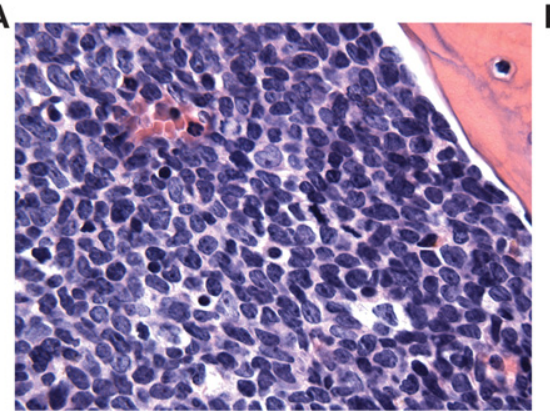

B

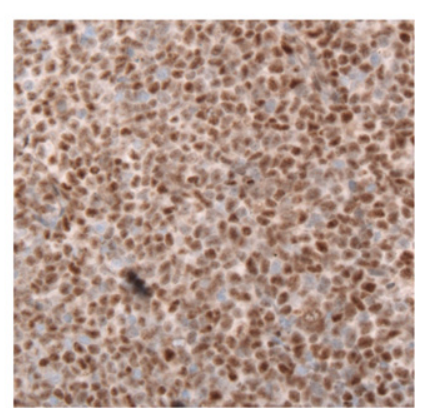

Figure 5. Characterization of ETP-ALL grafts in NSG-S mice (NSG mice expressing IL-3, GM-CSF, and KIT ligand). (A) Bone marrow histology (hematoxylin and eosin staining, 400× magnification). (B) Immunohistochemical staining for activated NOTCH1 (ICN1). Brown nuclear color denotes specific staining; hematoxylin was used as a counterstain.

CD45(dim), CD34+, HLA-DR+, CD3+, CD5+, CD7+, CD13+, and CD33+. Unfortunately, the leukemic blasts failed to grow when reinjected into secondary recipient animals, precluding further analysis.

\section{DISCUSSION}

Increasing attention is being paid to so-called "super-responders," outlier cases identified within the context of clinical trials in which tumors demonstrate profound responses to targeted therapies (Mehra et al. 2015). We report one such case, a relapsed refractory ETPALL arising out of a background of clonal hematopoiesis that demonstrated a complete response to the GSI BMS-906024. To our knowledge, this is the first patient with ETP-ALL who has been treated with a GSI. ETP-ALL is generally been considered to have a poorer prognosis than other T-ALL subtypes, and relapsed refractory disease is rarely curable, even with stem cell transplantation, highlighting the exceptional nature of this case.

Because we were unable to establish a stable patient-derived xenograft from this tumor, we can only speculate on the basis for the response of this tumor to BMS-906024. Although $\gamma$-secretase cleaves multiple surface receptors, the presence of a GSI-targetable NOTCH1 gain-of-function mutant associated with high levels of activated NOTCH1 in the leukemic blasts makes it likely that inhibition of Notch signaling was at least partially culpable for the response to BMS-906024 treatment. It has been reported that Notch signaling renders T-ALL cells resistant to killing by glucocorticoids and conversely that GSIs sensitize T-ALL cells to glucocorticoids (Deftos et al. 1998; Real et al. 2009). It is worth considering if this interaction might have contributed to the extraordinary responsiveness of this case of ETPALL, given that the first doses of BMS-906024 in cycles 1 and 2 were given along with $4 \mathrm{~d}$ of dexamethasone. We cannot exclude this possibility, but in short-term culture assays we did not observe any increased killing of patient blasts by dexamethasone in the presence of GSI (data not shown). We also note that following initiation of BMS-906024 blasts disappeared from the peripheral blood gradually over a period of 2-3 wk, without evidence of tumor lysis. Alternatively, HES1, the most sensitive gene to Notch inhibition in this patient's blasts, has been implicated in maintenance of stemness in a number of cancers (Liu et al. 2015), suggesting that its downregulation, alone or in combination with other targets such as HES4, might have led to clearance of leukemic blasts through differentiation. Study of additional responsive cases and establishment of mouse models will be needed to distinguish among these possibilities. 
Unexpectedly, one Notch target gene that is strongly correlated with response to Notch inhibition in cortical T-ALL, MYC, was not under the control of Notch in this tumor. Lack of response of MYC to Notch inhibition can be explained by the inactivate state of the Notch-dependent MYC long-range enhancer (NDME) in this tumor, which appears instead to rely on a BRD4-dependent long-range enhancer (BDME) defined previously in AML (Shi et al. 2013). It will be of interest to determine if ETP-ALL generally relies on the BDME to drive MYC, as this would provide a rationale for treatment of ETP-ALL will BRD4 inhibitors, which are now being tested in clinical trials.

The other oncogenic driver mutations in this case also merit comment. The CSF3R and PTPN11 gain-of-function mutations are in line with other studies showing that mutations in cytokine and growth factor signaling pathways are frequently observed in ETP-ALL (Coustan-Smith et al. 2009; Neumann et al. 2013). CSF3R mutations are associated with response of chronic neutrophilic leukemia to JAK inhibitors such as ruxilitinib and provide another possible avenue for targeted therapy in ETP-ALL. One other recently reported ETPALL associated with the CSF3R 1618T mutation (Maxson et al. 2013) also had a NOTCH1 NRR gain-of-function mutation (B Tyner, pers. comm.), indicating that this is a recurrent combination of mutations in this leukemia type. Also remarkable is the DNMT3A loss-of-function mutation, which was present in the ETP-ALL at homozygous dosage and persisted in the remission marrow at heterozygous dosage. The latter observation strongly suggests that this patient's ETP-ALL arose out of a background of clonal hematopoiesis, a common condition in older adults (Genovese et al. 2014; Jaiswal et al. 2014).

Unregulated Notch signaling drives normal hematopoietic stem cells to the CD4/CD8 double-positive stage of T-cell development (Pui et al. 1999); in contrast, Notch-mutated ETP-ALL cells, as in our case, arrest at what appears to correspond to an earlier stage of T-cell development during which cells maintain a potential for myeloid fate. We speculate that the inability of Notch to drive ETP-ALL cells to later stages of T-cell development stems from preexistence mutations (e.g., DNMT3A mutations) that skew the potential of hematopoietic stem cells toward myeloid fate. This may also explain the difference in response to Notch inhibition, as ETP-ALL cells represent a different cellular context than cortical-type T-ALL cells, and Notch effects are highly context dependent. Tests of this idea await development of genetically engineered mouse models with the combination of driver genes observed in this case.

\section{METHODS}

\section{Chromatin Immunoprecipitation (ChIP)}

ChIP was performed as described by Wang and colleagues (Wang et al. 2014) with the following modifications. Chromatin from formaldehyde-fixed cells $\left(5 \times 10^{6}\right.$ cells per histone mark) was fragmented to a size range of 200-700 bases with a Branson 250 Sonifier. Solubilized chromatin was immunoprecipitated with an antibody against H3K27ac $(2.5 \mu \mathrm{L}$; ActiveMotif, ab4729), and enrichment for H3K27ac was confirmed by dot blot as described (Ernst et al. 2011). Antibody-chromatin complexes were pulled down with protein $G$ magnetic beads (Dynabead, 10003D), washed and then eluted. After cross-link reversal and proteinase $\mathrm{K}$ treatment, immunoprecipitated DNA was treated with RNase and purified with Agencourt AMPure XP (Beckman Coulter A63880). Libraries were prepared according to Illumina's instructions. ChIP DNA and input controls were sequenced with the Illumina HiSeq 2500 instrument. ChIP-seq data were processed and analyzed as described (Wang et al. 2014). Briefly, sequence reads were mapped to human genome build hg38 using Bowtie 2 (Langmead and Salzberg 2012). Uniquely mapped sequence reads were retained (Table 3). Genomic enrichment was identified using MACS 2.0 with 


\begin{tabular}{lllrr}
\hline Table 3. Summary of sequencing studies & \multicolumn{1}{c}{} \\
\hline Sample & \multicolumn{1}{c}{ Sequencing type } & $\begin{array}{r}\text { Total } \\
\text { reads }\end{array}$ & $\begin{array}{c}\text { Aligned } \\
\text { reads (\%) }\end{array}$ & $\begin{array}{r}\text { Average } \\
\text { coverage }\end{array}$ \\
\hline Aspirated marrow at presentation & Targeted-exon sequencing & $1.6 \times 10^{7}$ & 96.0 & 175 -fold \\
Aspirated marrow at presentation & Whole-exon sequencing & $1.3 \times 10^{9}$ & 90.9 & $90-$-fold \\
Aspirated marrow at remission & Whole-exon sequencing & $9.1 \times 10^{8}$ & 91.7 & $124-$-fold \\
Blasts at presentation treated with DMSO & RNA-seq & $1.3 \times 10^{9}$ & 65.4 & NA \\
Blasts at presentation treated with GSI & RNA-seq & $7.9 \times 10^{9}$ & 67.0 & NA \\
Blasts at presentation & H3K27ac ChIP-seq & $8.1 \times 10^{8}$ & 98.0 & NA \\
\hline
\end{tabular}

DMSO, dimethyl sulfoxide; RNA-seq, RNA sequencing; GSI, $\gamma$-secretase inhibitor; ChIP-seq, chromatin immunoprecipitation sequencing; NA, not applicable.

broad peak calling under a false discovery threshold of 0.01 (Zhang et al. 2008). ChIP-seq traces were produced using the R package Gvis.

\section{Targeted Exome Sequencing (TES) and Whole-Exome Sequencing (WES)}

TES was performed as described (Wagle et al. 2012). WES was performed using DNA extracted from peripheral blood mononuclear cell pellets. Genomic DNA (250 ng) was subjected to shearing, barcoded sequencing library preparation, and hybrid capture as described (Fisher et al. 2011). Libraries were sequenced on the Broad Sequencing Platform (Cambridge, MA) with an Illumina HiSeq 2000 (San Diego, CA) using 76-bp paired-end reads. Sequencing data were aligned to the human reference genome hg19 using Picard tools (http://broadinstitute.github.io/picard/). Mutation calls were made on the WES samples with the mutation calling workflow in Firehose (https://www.broadinstitute.org/cancer/ cga/Firehose) using Mutect (Cibulskis et al. 2013) with default parameters and enabled to run without matched normal DNA. Each mutation call made by Mutect was annotated using Oncotator (Ramos et al. 2015). Mutations that differed between the diagnostic and remission samples were curated from these lists. Likely driver mutations in NOTCH1, PTPN11, DNMT3A, and CSF3R were validated by aligning the original BAM files to hg19 and visualizing aligned reads in IGV (Robinson et al. 2011).

\section{Sanger Sequencing}

Sanger sequencing of the region containing the DNMT3A c.1204C > T substitution was performed after PCR amplification of genomic DNA obtained from leukemic and remission marrow and from saliva following allogeneic hematopoietic stem cell transplantation using PFU Turbo DNA polymerase (Stratagene) with $100 \mu \mathrm{M}$ of each primer in the presence of $10 \%$ DMSO under standard buffer and $\mathrm{MgCl}_{2}$ conditions. PCR conditions were 30 cycles of $95^{\circ} \mathrm{C} \times 1 \mathrm{~min}, 55^{\circ} \mathrm{C} \times 2 \mathrm{~min}$, and $72^{\circ} \mathrm{C} \times 3 \mathrm{~min}$. PCR amplification products were sequenced directly by GeneWiz. PCR primers are given in Supplemental Table 1.

\section{RNA Sequencing (RNA-seq)}

RNA-seq was performed on poly(A) selected RNA extracted from $1 \times 10^{6}$ cultured leukemic cells (see below). Complementary DNA synthesis, barcoded library preparation and sequencing were performed as described (Wagle et al. 2014). RNA-seq data were analyzed using the PRADA pipeline as described (Bambury et al. 2015). Resultant data were transformed into gene-level expression values for downstream analysis (DeLuca et al. 2012). 


\section{Cell Culture and Cell Culture Assays}

Mononuclear cells were isolated from marrow using Ficoll gradients, washed, and resuspended in WIT medium in the presence of MS5 feeder cells expressing the Notch ligand DLL-1 for short-term culture assays, as described (Yost et al. 2013). To study effects of Notch inhibition, cultures were treated with the GSI DBZ (dibenzazepine, $1 \mu \mathrm{M}$ ) or DMSO vehicle (control). Notch reporter gene assays were performed in U2OS cells as described (Malecki et al. 2006). Antibody against $\beta$-actin was obtained from Sigma-Aldrich (A1978), and antibodies against activated NOTCH1 (D3B8) and MYC (9402) were from Cell Signaling Technologies.

\section{Mouse Xenograft Studies}

Mononuclear cells from bone marrow aspirate were isolated on Ficoll gradients and injected by tail vein into NOD scid IL2R $\gamma$ chain knockout (NSG) and NSG mice expressing IL-3, GM-CSF, and KIT ligand (NSG-S). Mice were followed for development of leukemia by monitoring the peripheral blood for appearance of blasts expressing human CD45. Immunohistochemical stains for activated $\mathrm{NOTCH} 1$ was performed in formalin-fixed paraffin-embedded sections as described (Kluk et al. 2013). All animal studies were performed using protocols approved by the Columbia University Medical Center IACUC.

\section{ADDITIONAL INFORMATION}

\section{Ethics Statement}

Human marrow, peripheral blood, and saliva samples were collected with informed consent and downstream analyses were performed under protocols 01-206 and 11-204 that were approved by the Institutional Review Board of the Dana-Farber/Harvard Cancer Center.

\section{Database Deposition and Access}

Whole-exome sequencing, RNA sequencing, and ChIP-seq data are available through the NCBI Gene Expression Omnibus (GEO; http://www.ncbi.nlm.nih.gov/geo/) under accession number GSE71414. Point substitution variants were submitted to ClinVar (http://www.ncbi. nlm.nih.gov/clinvar/) (accession numbers SCV000239862-SCV000239865).

\section{Author Contributions}

B.K., A.B., C.S.P., E.S., F.C.K., and M.M. contributed to analysis and interpretation of nextgeneration sequencing data. A.G. performed and interpreted TCR $\gamma$ gene studies. D.M.D. interpreted flow cytometric findings. M.K. performed and interpreted immunohistochemical studies. L.P. performed and interpreted ex vivo assays using clinical samples and assays of the functional consequences of NOTCH1 mutations. S.C.B. provided structural models of the negative regulatory region of NOTCH1. A.L.K. oversaw xenograft studies. P.Z.-M. and D.J.D. contributed and reviewed the clinical features of the case. J.C.A. contributed micrographs of primary tumor specimens and xenografted tumors. All authors contributed to the writing of the manuscript.

Competing Interest Statement

The authors have declared no competing interest.

Received June 27, 2015; accepted in revised form July 25 , 2015.

\section{Funding}

J.C.A. is supported by a grant from the National Institutes of Health (P01CA119070), a Leukemia \& Lymphoma Society Specialized Center of Research (LLS SCOR) grant, and a grant from the William Lawrence and Blanche Hughes Foundation. 


\section{REFERENCES}

Aster JC, Blacklow SC. 2012. Targeting the Notch pathway: twists and turns on the road to rational therapeutics. J Clin Oncol 30: 2418-2420.

Bambury RM, Bhatt AS, Riester M, Pedamallu CS, Duke F, Bellmunt J, Stack EC, Werner L, Park R, lyer G, et al. 2015. DNA copy number analysis of metastatic urothelial carcinoma with comparison to primary tumors. BMC Cancer 15: 242.

Cibulskis K, Lawrence MS, Carter SL, Sivachenko A, Jaffe D, Sougnez C, Gabriel S, Meyerson M, Lander ES, Getz G. 2013. Sensitive detection of somatic point mutations in impure and heterogeneous cancer samples. Nat Biotechnol 31: 213-219.

Coustan-Smith E, Mullighan CG, Onciu M, Behm FG, Raimondi SC, Pei D, Cheng C, Su X, Rubnitz JE, Basso G, et al. 2009. Early T-cell precursor leukaemia: a subtype of very high-risk acute lymphoblastic leukaemia. Lancet Oncol 10: 147-156.

Deftos ML, He YW, Ojala EW, Bevan MJ. 1998. Correlating notch signaling with thymocyte maturation. Immunity 9: 777-786.

DeLuca DS, Levin JZ, Sivachenko A, Fennell T, Nazaire MD, Williams C, Reich M, Winckler W, Getz G. 2012. RNA-SeQC: RNA-seq metrics for quality control and process optimization. Bioinformatics 28: $1530-1532$

Ernst J, Kheradpour P, Mikkelsen TS, Shoresh N, Ward LD, Epstein CB, Zhang X, Wang L, Issner R, Coyne $M$, et al. 2011. Mapping and analysis of chromatin state dynamics in nine human cell types. Nature 473: 43-49.

Fisher S, Barry A, Abreu J, Minie B, Nolan J, Delorey TM, Young G, Fennell TJ, Allen A, Ambrogio L, et al. 2011. A scalable, fully automated process for construction of sequence-ready human exome targeted capture libraries. Genome Biol 12: R1.

Genovese G, Kahler AK, Handsaker RE, Lindberg J, Rose SA, Bakhoum SF, Chambert K, Mick E, Neale BM, Fromer $\mathrm{M}$, et al. 2014. Clonal hematopoiesis and blood-cancer risk inferred from blood DNA sequence. N Engl J Med 371: 2477-2487.

Gordon WR, Roy M, Vardar-Ulu D, Garfinkel M, Mansour MR, Aster JC, Blacklow SC. 2009a. Structure of the Notch1-negative regulatory region: implications for normal activation and pathogenic signaling in T-ALL. Blood 113: 4381-4390.

Gordon WR, Vardar-Ulu D, L'Heureux S, Ashworth T, Malecki MJ, Sanchez-Irizarry C, McArthur DG, Histen G, Mitchell JL, Aster JC, et al. 2009b. Effects of S1 cleavage on the structure, surface export, and signaling activity of human Notch1 and Notch2. PLoS One 4: e6613.

Gutierrez A, Dahlberg SE, Neuberg DS, Zhang J, Grebliunaite R, Sanda T, Protopopov A, Tosello V, Kutok J, Larson RS, et al. 2010. Absence of biallelic TCR $\gamma$ deletion predicts early treatment failure in pediatric T-cell acute lymphoblastic leukemia. J Clin Oncol 28: 3816-3823.

Herranz D, Ambesi-Impiombato A, Palomero T, Schnell SA, Belver L, Wendorff AA, Xu L, Castillo-Martin M, Llobet-Navas D, Cordon-Cardo C, et al. 2014. A NOTCH1-driven MYC enhancer promotes T cell development, transformation and acute lymphoblastic leukemia. Nat Med 20: 1130-1137.

Jaiswal S, Fontanillas P, Flannick J, Manning A, Grauman PV, Mar BG, Lindsley RC, Mermel CH, Burtt N, Chavez A, et al. 2014. Age-related clonal hematopoiesis associated with adverse outcomes. N Engl J Med 371: 2488-2498.

Kluk MJ, Ashworth T, Wang H, Knoechel B, Mason EF, Morgan EA, Dorfman D, Pinkus G, Weigert O, Hornick JL, et al. 2013. Gauging NOTCH1 activation in cancer using immunohistochemistry. PLoS One 8: e67306.

Kopan R, Ilagan MX. 2009. The canonical Notch signaling pathway: unfolding the activation mechanism. Cell 137: $216-233$.

Langmead B, Salzberg S. 2012. Fast gapped-read alignment with Bowtie 2. Nat Methods 9: 357-359.

Larson RA, Dodge RK, Linker CA, Stone RM, Powell BL, Lee EJ, Schulman P, Davey FR, Frankel SR, Bloomfield CD, et al. 1998. A randomized controlled trial of filgrastim during remission induction and consolidation chemotherapy for adults with acute lymphoblastic leukemia: CALGB study 9111. Blood 92: 1556-1564.

Liu ZH, Dai XM, Du B. 2015. Hes1: a key role in stemness, metastasis and multidrug resistance. Cancer Biol Ther 16: 353-359.

Malecki MJ, Sanchez-Irizarry C, Mitchell JL, Histen G, Xu ML, Aster JC, Blacklow SC. 2006. Leukemia-associated mutations within the NOTCH1 heterodimerization domain fall into at least two distinct mechanistic classes. Mol Cell Biol 26: 4642-4651.

Maxson JE, Gotlib J, Pollyea DA, Fleischman AG, Agarwal A, Eide CA, Bottomly D, Wilmot B, McWeeney SK, Tognon CE, et al. 2013. Oncogenic CSF3R mutations in chronic neutrophilic leukemia and atypical CML. N Engl J Med 368: 1781-1790. 
Mehra N, Lorente D, de Bono JS. 2015. What have we learned from exceptional tumour responses?: review and perspectives. Curr Opin Oncol 27: 267-275.

Neumann M, Heesch S, Schlee C, Schwartz S, Gokbuget N, Hoelzer D, Konstandin NP, Ksienzyk B, Vosberg S, Graf A, et al. 2013. Whole-exome sequencing in adult ETP-ALL reveals a high rate of DNMT3A mutations. Blood 121: 4749-4752.

Pui JC, Allman D, Xu L, DeRocco S, Karnell FG, Bakkour S, Lee JY, Kadesch T, Hardy RR, Aster JC, et al. 1999. Notch1 expression in early lymphopoiesis influences B versus $T$ lineage determination. Immunity 11: 299-308.

Ramos AH, Lichtenstein L, Gupta M, Lawrence MS, Pugh TJ, Saksena G, Meyerson M, Getz G. 2015. Oncotator: cancer variant annotation tool. Hum Mutat 36: E2423-E2429.

Real PJ, Tosello V, Palomero T, Castillo M, Hernando E, de Stanchina E, Sulis ML, Barnes K, Sawai C, Homminga I, et al. 2009. $\gamma$-Secretase inhibitors reverse glucocorticoid resistance in T cell acute lymphoblastic leukemia. Nat Med 15: 50-58.

Robinson JT, Thorvaldsdottir H, Winckler W, Guttman M, Lander ES, Getz G, Mesirov JP. 2011. Integrative genomics viewer. Nat Biotechnol 29: 24-26.

Shi J, Whyte WA, Zepeda-Mendoza CJ, Milazzo JP, Shen C, Roe JS, Minder JL, Mercan F, Wang E, EckersleyMaslin MA, et al. 2013. Role of SWI/SNF in acute leukemia maintenance and enhancer-mediated Myc regulation. Genes Dev 27: 2648-2662.

Tartaglia M, Gelb BD. 2005. Noonan syndrome and related disorders: genetics and pathogenesis. Annu Rev Genomics Hum Genet 6: 45-68.

Van Vlierberghe P, Ferrando A. 2012. The molecular basis of T cell acute lymphoblastic leukemia. J Clin Invest 122: 3398-3406.

van Vlierberghe P, Meijerink JP, Lee C, Ferrando AA, Look AT, van Wering ER, Beverloo HB, Aster JC, Pieters R. 2006. A new recurrent $9 q 34$ duplication in pediatric T-cell acute lymphoblastic leukemia. Leukemia 20: 1245-1253.

Wagle N, Berger MF, Davis MJ, Blumenstiel B, Defelice M, Pochanard P, Ducar M, Van Hummelen P, Macconaill LE, Hahn WC, et al. 2012. High-throughput detection of actionable genomic alterations in clinical tumor samples by targeted, massively parallel sequencing. Cancer Discov 2: 82-93.

Wagle N, Van Allen EM, Treacy DJ, Frederick DT, Cooper ZA, Taylor-Weiner A, Rosenberg M, Goetz EM, Sullivan RJ, Farlow DN, et al. 2014. MAP kinase pathway alterations in BRAF-mutant melanoma patients with acquired resistance to combined RAF/MEK inhibition. Cancer Discov 4: 61-68.

Wang H, Zang C, Taing L, Arnett KL, Wong YJ, Pear WS, Blacklow SC, Liu XS, Aster JC. 2014. NOTCH1-RBPJ complexes drive target gene expression through dynamic interactions with superenhancers. Proc Nat Acad Sci 111: 705-710.

Yashiro-Ohtani Y, Wang H, Zang C, Arnett KL, Bailis W, Ho Y, Knoechel B, Lanauze C, Louis L, Forsyth KS, et al. 2014. Long-range enhancer activity determines Myc sensitivity to Notch inhibitors in T cell leukemia. Proc Natl Acad Sci 111: E4946-E4953.

Yost AJ, Shevchuk OO, Gooch R, Gusscott S, You MJ, Ince TA, Aster JC, Weng AP. 2013. Defined, serum-free conditions for in vitro culture of primary human T-ALL blasts. Leukemia 27: 1437-1440.

Zhang Y, Liu T, Meyer CA, Eeckhoute J, Johnson DS, Bernstein BE, Nusbaum C, Myers RM, Brown M, Li W, et al. 2008. Model-based analysis of ChIP-Seq (MACS). Genome Biol 9: R137.

Zhang J, Ding L, Holmfeldt L, Wu G, Heatley SL, Payne-Turner D, Easton J, Chen X, Wang J, Rusch M, et al 2012. The genetic basis of early T-cell precursor acute lymphoblastic leukaemia. Nature 481: 157-163. 


\section{COLD SPRING HARBOR Molecular Case Studies}

\section{Complete hematologic response of early T-cell progenitor acute lymphoblastic leukemia to the $\gamma$-secretase inhibitor BMS-906024: genetic and epigenetic findings in an outlier case}

Birgit Knoechel, Ami Bhatt, Li Pan, et al.

Cold Spring Harb Mol Case Stud 2015, 1: a000539

Access the most recent version at doi:10.1101/mcs.a000539
Supplementary http://molecularcasestudies.cshlp.org/content/suppl/2015/09/03/1.1.a000539.DC Material 1
References This article cites 38 articles, 11 of which can be accessed free at: http://molecularcasestudies.cshlp.org/content/1/1/a000539.full.html\#ref-list-1
License This article is distributed under the terms of the Creative Commons Attribution License, which permits unrestricted reuse and redistribution provided that the original author and source are credited.
Email Alerting Receive free email alerts when new articles cite this article - sign up in the box at the Service top right corner of the article or click here.

